\title{
Dynamical Variation of Plasmasphere Revealed by PWS Data onboard the Akebono (EXOS-D) Satellite
}

\author{
Hiroshi OYA \\ Department of Astronomy and Geophysics, Tohoku University, Sendai 980-8578, Japan
}

(Received February 28, 1996; Revised September 20, 1996; Accepted November 10, 1997)

\begin{abstract}
From the upper hybrid frequency data observed by PWS onboard the Akebono satellite, the entire plasma density profile along the satellite paths have been obtained being based on the plasma neutrality condition. The analyses of the plasma density deduced from the upper hybrid frequency reveal the dynamical variation of the plasmaspheric plasma density profile (PPDP). The PPDP variation is especially characterized by the intimate correspondence to the time variation of $D s t$ that indicates the time derivative-coefficient larger than $5 \mathrm{nT} /$ hour. Variation phenomena of PPDP are divided into two categories depending on the phases of $D s t$ variations; these are the phenomena which occur in the phase of the positive time derivative-coefficient (PDC) of Dst; and the phenomena which occur in the phase of the negative time derivative-coefficient (NDC) of Dst. Both in PDC, and NDC phases, the plasmapause structures show the sharpening of their cliff feature of the plasma density distribution, but a few hours later, all these traditionally convinced enhancement effects of the plasma convection in the neighboring plasma sheet regions are taken over by the effects of plasmapause disruption without depending on the local time of the consideration. These plasmapause disruptions are caused by the exodus of the plasmaspheric plasma in the NDC phase while the hot plasma sheet plasma immigrates into the plasmasphere in the phase of PDC. Because of extreme inhomogeneity of the plasma temperature between the original plasmaspheric warm plasma and hot plasma from the plasma sheet region, there exist sharp discontinuous boundaries across the magnetic field lines in the plasmasphere. The evidence has been detected as the "donkey ears" phenomena by the observation of PWS onboard the Akebono satellite. It is disclosed that the exodus of plasma from the plasmasphere in NDC phase of $D s t$, and the immigration of plasma into the plasmasphere in PDC phase are caused by $\boldsymbol{E} \times \boldsymbol{B}$ drift due to the induction electric field that is strictly related to $\partial B / \partial t$. The arrival of the front of the injected hot plasma delays with times ranging from $15 \mathrm{~h}$ to $2.5 \mathrm{~h}$ corresponding to the time derivative-coefficient of $D s t$, respectively from $5 \mathrm{nT} / \mathrm{hour}$ to $30 \mathrm{nT} /$ hour. As higher the time derivative-coefficient becomes, the faster the drift velocity is increased; i.e., the delay time of arrival of the hot plasma front from the original plasmapause position to the satellite level becomes short for the fast $\boldsymbol{E} \times \boldsymbol{B}$ drift.
\end{abstract}

\section{Introduction}

After the establishment of the definition of the plasmasphere being related to the discovery of the plasmapause by Carpenter (1963) and followed confirmation by the satellite observations (Chappel et al., 1970), the concept of the plasmasphere is established as the region smoothly connected to the topside ionosphere by diffusive equilibrium processes of the plasma along the corresponding magnetic flux tube (Angerami and Thomas, 1964).

The plasmapause which separates the plasmasphere from the plasma sheet in the inner magnetosphere is thought to be formed due to the remarkable differentiation of the convection of the inner magnetosphere plasma between co-rotation region of the plasma, as the concept was initially proposed by Nishida (1966) based on the Axford-Hines magnetospheric plasma convection model (1961); the model has been further improved to meet more realistic magnetospheric plasma condition in which the geomagnetic tail effects are included (Brice, 1967). Studies on all of these concepts for plasmasphere and plasmapause are now entering in the era when the concept is evolutionary rewritten by recent discoveries of the plasmasphere 
and plasmapause behaviors. The data of plasma density obtained by the PWS (Plasma Wave and Sounder Experiment, Oya et al., 1990) onboard the Akebono satellite have provided significant evidences of the plasma density variations in the plasmasphere and plasmapause in the various conditions of the magnetic field activities including quiet and storm times. During the period of the magnetic storm, the occurrence of the disruption of the plasmapause has been confirmed both in the main phase and the recovery phase of the large magnetic storms (Oya, 1991). Especially characteristic depression of the plasma density takes place centered around the magnetic equator in the recovery phase of the magnetic storms; the plasma density depression phenomena in the recovery phase of the magnetic storm is named "Donkey Ears Phenomena" due to its apparent shape in the plasma density diagram where the electron density plotted show two maxima along the satellite paths which pass across plasmasphere through the region of the magnetic equator (Oya, 1991). The purpose of the present paper is to describe the dynamical features of plasmasphere phenomena associated with the storm time magnetic field variation and to propose the origin of the dynamical plasma motion across the magnetic field as the results of the plasma drift caused by the time dependent electric field which is generated by the time varying effects of the ring current. The effect is called "Betatron drift" in this paper.

\section{Typical Plasmasphere Behaviors Detected by PWS onboard the Akebono (EXOS-D) Satellite}

\subsection{Main phase phenomena}

The case of plasmapause destruction has been reported being based on the plasma density data obtained by PWS onboard the Akebono satellite; the essential part is reproduced in Figs. 1(A) to 1(C). In addition to these remarkable results, the results of our recent investigations have also indicated the occurrence of apparent disruptions of the plasmapause with reduction of the plasma density inside the plasmasphere, as has been given in Fig. 2, during the storm time which had occurred from March 13 to March 15. Because of the asymmetry of the orbit with respect to the magnetic equator, the feature of the plasmapause is not indicated as the case of observation results along the satellite paths during the special period when the apogee is near the equator forming approximately symmetrical orbit with respect to the magnetic equator; the plasmapause structure is revealed as the plasma trough in the topside ionosphere in the northern hemisphere, in the low altitude, while the plasmapause shows clear feature in the southern hemisphere in the extremely asymmetrical satellite orbit. All these features of the plasmapause however disappear during the main phase of the magnetic storms without the local time dependence suggesting outward flow of the plasma from the inside plasmasphere into the plasma sheet outside of the plasmapause.

\section{2 "Donkey ears" phenomena in the recovery phase of the magnetic storms}

A unique feature of the plasma density profile has frequently been observed by PWS onboard the Akebono (EXOS-D) satellite while the satellite passes through the plasmasphere across the magnetic equator in the recovery phase of the large magnetic storms. The characteristic feature of the profile of the plasma density distribution shows depression of the plasma density in a region centered around the magnetic equator while high density parts of the plasma density remain in the higher latitude regions continuously extended to the high latitude plasmapause. These remained high density parts show clear conjugacy in their related invariant magnetic latitude: the diagram of the plasma density shows, therefore, similar outlook of an animal head with fairly long ears. The phenomena are, then called "Donkey ears" (Oya, 1991). In Fig. 3, an example of the donkey ears phenomena is given for the dynamic spectra of the plasma wave observation by PWS where the upper hybrid wave emissions indicate relative feature of the plasma density variation (panel A); the obtained plasma density from the observed upper hybrid frequency is given in panel $\mathrm{B}$.

To investigate the occurrence condition of the donkey ears phenomena, we have randomly selected an interval of the observation of the Akebono satellite; for this interval the Dst value is plotted as given in Fig. 4. The periods when the donkey ears phenomena have occurred are indicated with arrows in this series of the Dst data. The result indicates that the donkey ears phenomena generally take place during the 


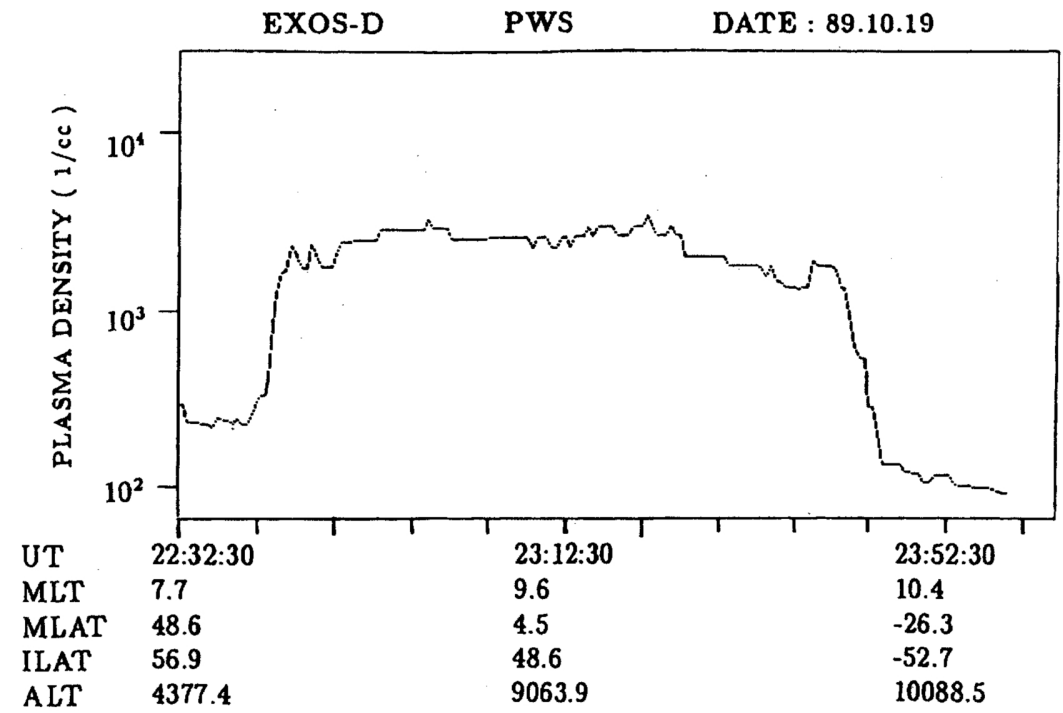

(A)

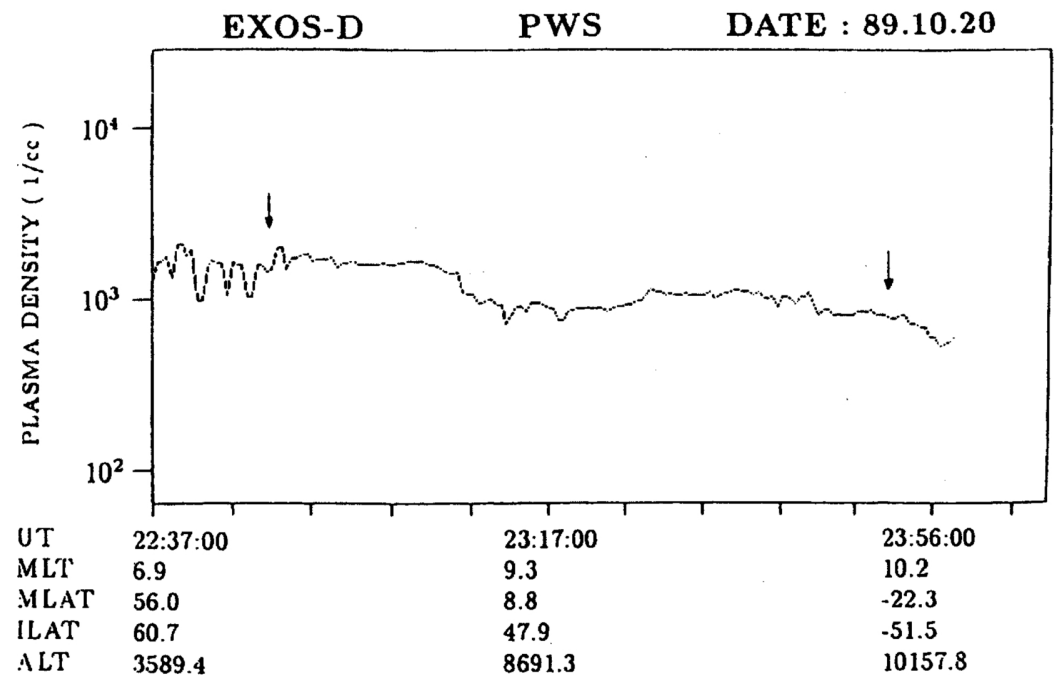

(B)

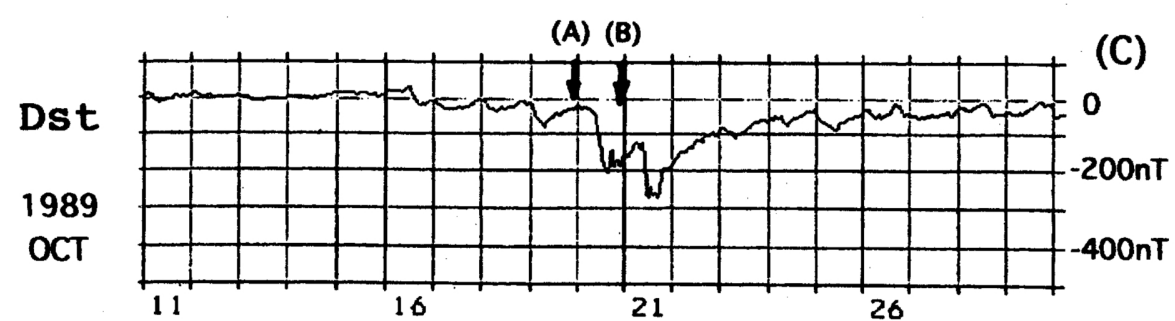

Fig. 1. Disruption of plasmapause suggesting the outflow of the plasma from the plasmasphere in the main phase of the large magnetic storm (after Oya, 1991). (A) Very early stage of the main phase where apparent plasmapause structure is indicated. (B) Intermediate stage of the main phase where the plasmapause structure has completely disappeared. (C) Dst diagram covering the period of the large magnetic storm including the main phase; allows (A) and (B) correspond to the period given in panels (A) and (B). 
( I )

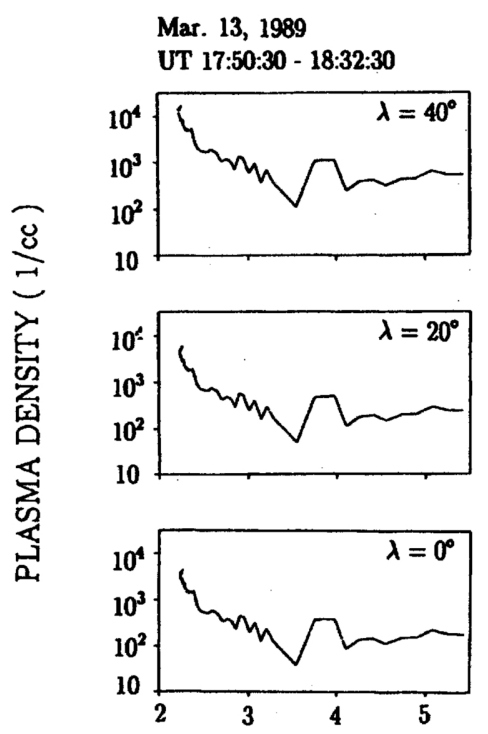

( II )

Mar. 14, 1989

UT 18:34:30 - 19:14:00
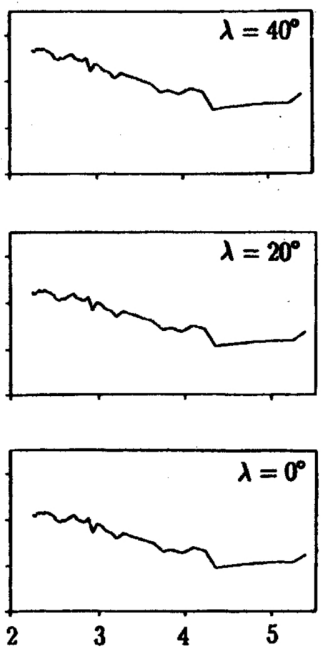

( III )

Mar. 15, 1989

UT 01:12:00 - 01:50:30
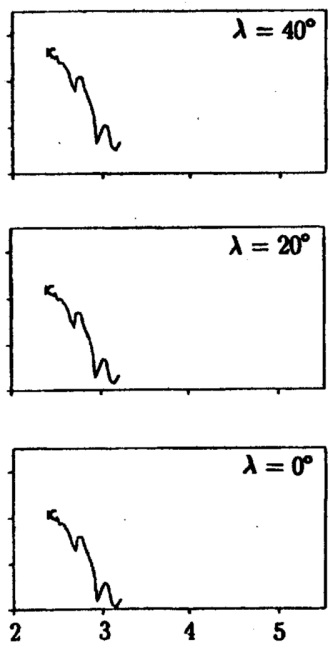

\section{L-VALUE}

(B)

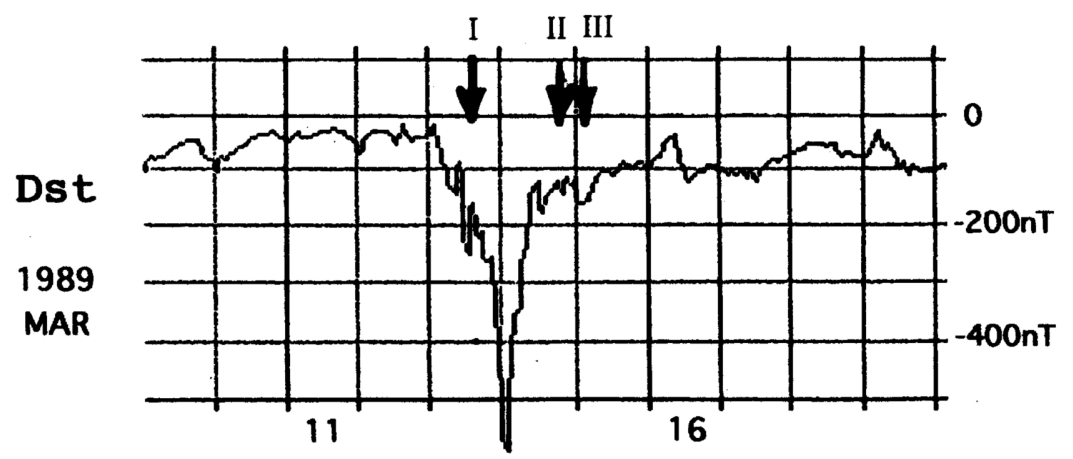

Fig. 2. (A) Plasma density profiles in the plasmasphere obtained by applying the diffusive equilibrium model starting from the observed plasma density along the EXOS-D orbit, for the period of the large magnetic storm of March 13-15, 1989. Plasma density profiles corresponding to the observations, (I) March 13, from 17:50:30 to 18:32:30 UT, (II) March 14, from 18:34:30 to 19:14:00 and (III) March 15, from 01:12:00 to 01:50:30 are plotted versus $L$ value with given magnetic latitude $\lambda=0^{\circ}$, $20^{\circ}$, and $40^{\circ}$ as parameter. (B) Dst diagram of March, 1989 covering the period of the large magnetic storm of March 1315. The periods, when the plasmaspheric plasma density profile are plotted, are indicated by corresponding symbols (I), (II) and (III).

recovery phase of magnetic storms.

To see a more detailed feature of time series variation in the forming processes of the donkey ears phenomena, we have selected an interval of the Akebono satellite data observed between December 4, 06:44UT to December 5, 05:48 UT in 1989, in Fig. 5 where the data of upper hybrid frequency in the dynamic spectra are indicated with the Dst values. We can see the progressing feature of the donkey ears in the periods given by panels 3 , and 4 given in Fig. 5 . Because of slow recovery of the magnetic field with 

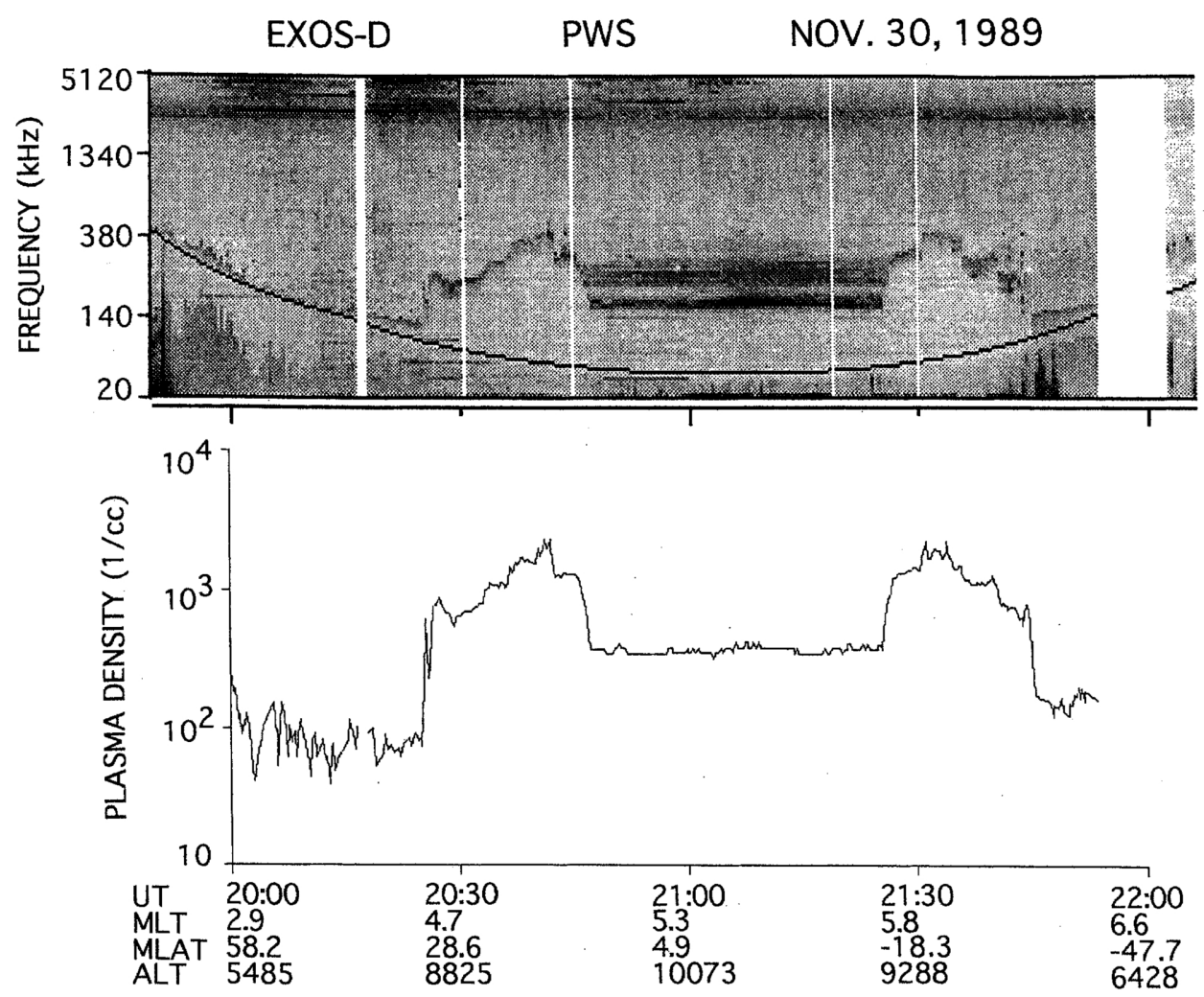

Fig. 3. Example of the donkey ears phenomena revealed by the upper hybrid wave observation of PWS onboard the Akebono (EXOS-D) satellite (A) and obtained electron density (B). The obtained electron density profile along the satellite path of which apogee is in the equator region around $10,000 \mathrm{~km}$, in this case, shows depression, around the equator region, by $1 / 5$ of the quiet time plasmasphere in the same region. There are shock like contact region with cold dense plasmaspheric plasma in the latitude around $47 \sim 8^{\circ}$ both in the northern and southern hemispheres. Because of shape of the plasma density profile along the approximately symmetrical satellite orbit with respect to the magnetic equator, the entire plasmaspheric plasma density profile in this period is called "donkey ears phenomena" (after Oya, 1991).

the rate of $5 \mathrm{nT} / \mathrm{H}$, the arrival of the depression effects at the satellite path at about $L=2.5$ is delayed about 10 hours. This delay time is however, independent to the characteristic time of the plasma density depletion effects. Once the phenomena of the donkey ears approach the level of the satellite path the density depression progresses with the characteristic time of 3 hours. When we see the panel 5 of Fig. 5 , the density of the entire plasmasphere is completely depleted to the level of $1 / 10$ of regular plasmaspheric plasma density distribution.

In this sequential data in Fig. 5, it is clearly indicated that the donkey ears phenomena appear as the result of the depression of the plasma density which start from the region of the magnetic equator and proceed to higher L-value regions. When the donkey ears phenomena proceed, the plasma density value at equator side in the plasmasphere shows extremely sharp gradient forming a shock like structure. It should be noted that the characteristic time of the evolution for forming the donkey ears structure that is defined as a period between the starting time and the most developed time of the phenomena is in the range from 2 to 3 hours. This characteristic time is almost one order of the magnitude shorter than the characteristic period for the magnetic field filling processes being fed from the ionosphere (Singh and Horwitz, 1992; and references in it). 


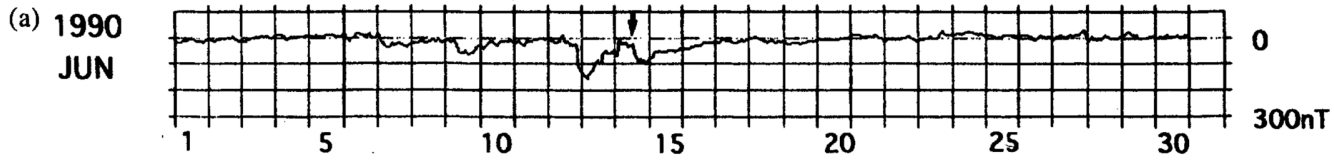

1990

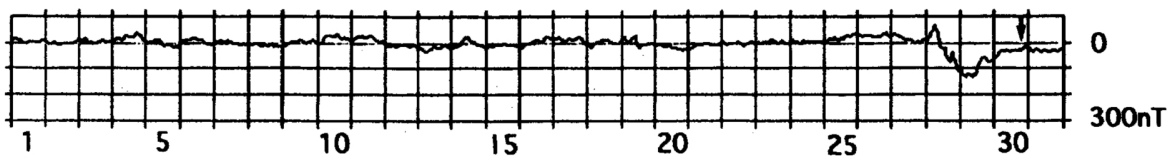

1990

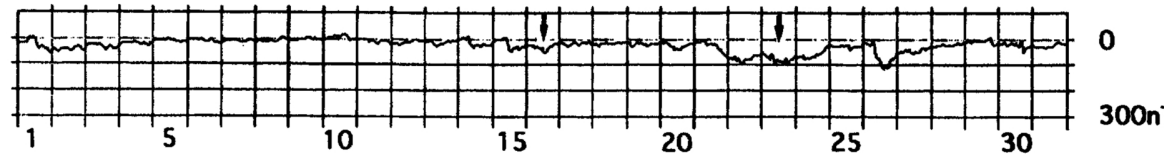

1990

SEP

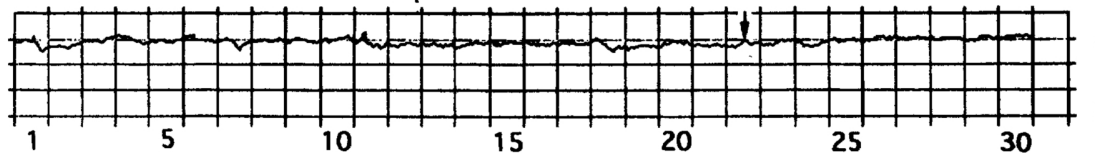

0

1990

OCT

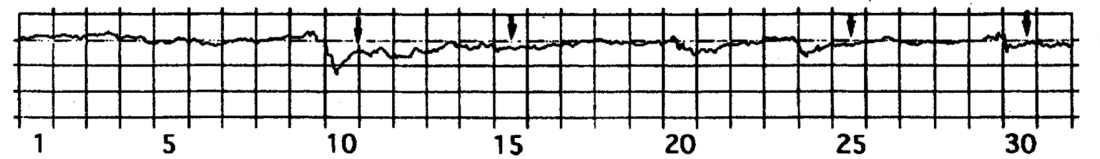

$300 \mathrm{nT}$

(b) 1991

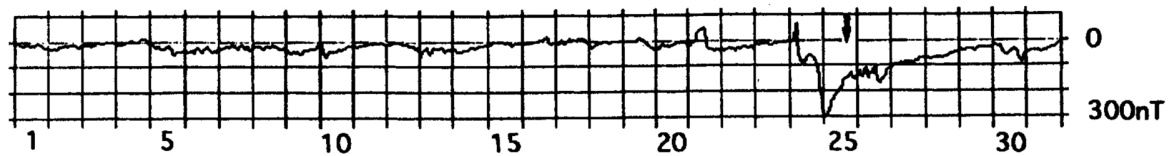

1991

APR

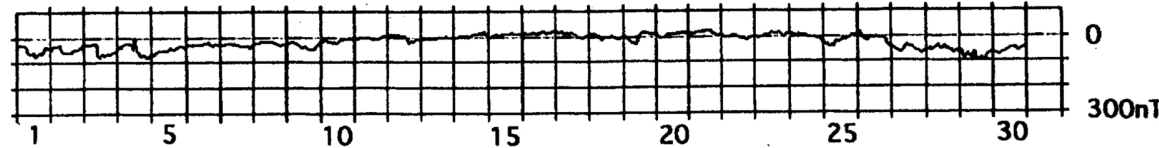

1991

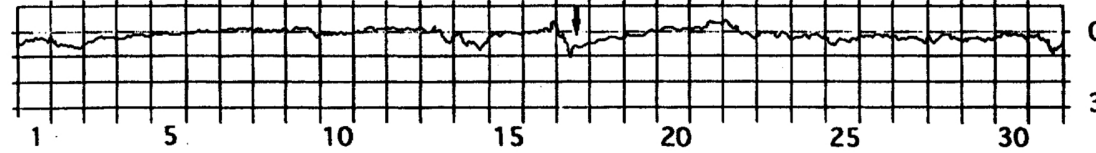

$300 \mathrm{nT}$

1991

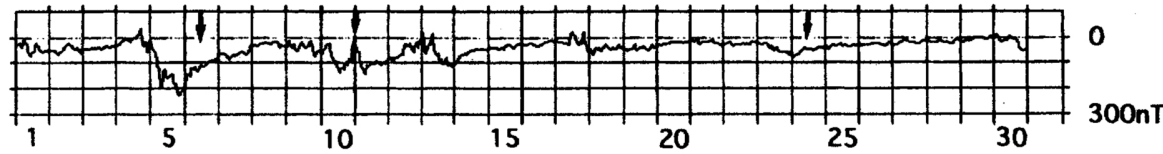

1991

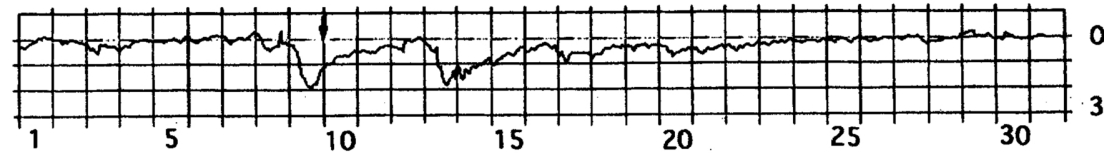

300nT

1991

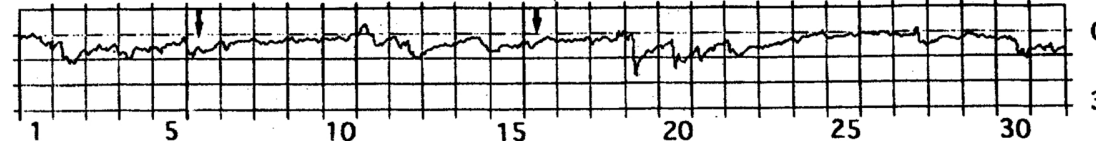

$300 \mathrm{nT}$

Fig. 4. The occurrence of the donkey ears phenomena in relation to Dst for a randomly picked up period (a) from June 1990 to Oct. 1990 and (b) from March 1991 to Aug. 1991. The selection of the donkey ears phenomena is only made for the data obtained from Kagoshima Space Center Japan. We should state, therefore, that the all donkey ears phenomena that we have investigated were always taking place in the period close to the recovery phase of the magnetic storms or the period close to $\partial D s t / \partial t>0$. 

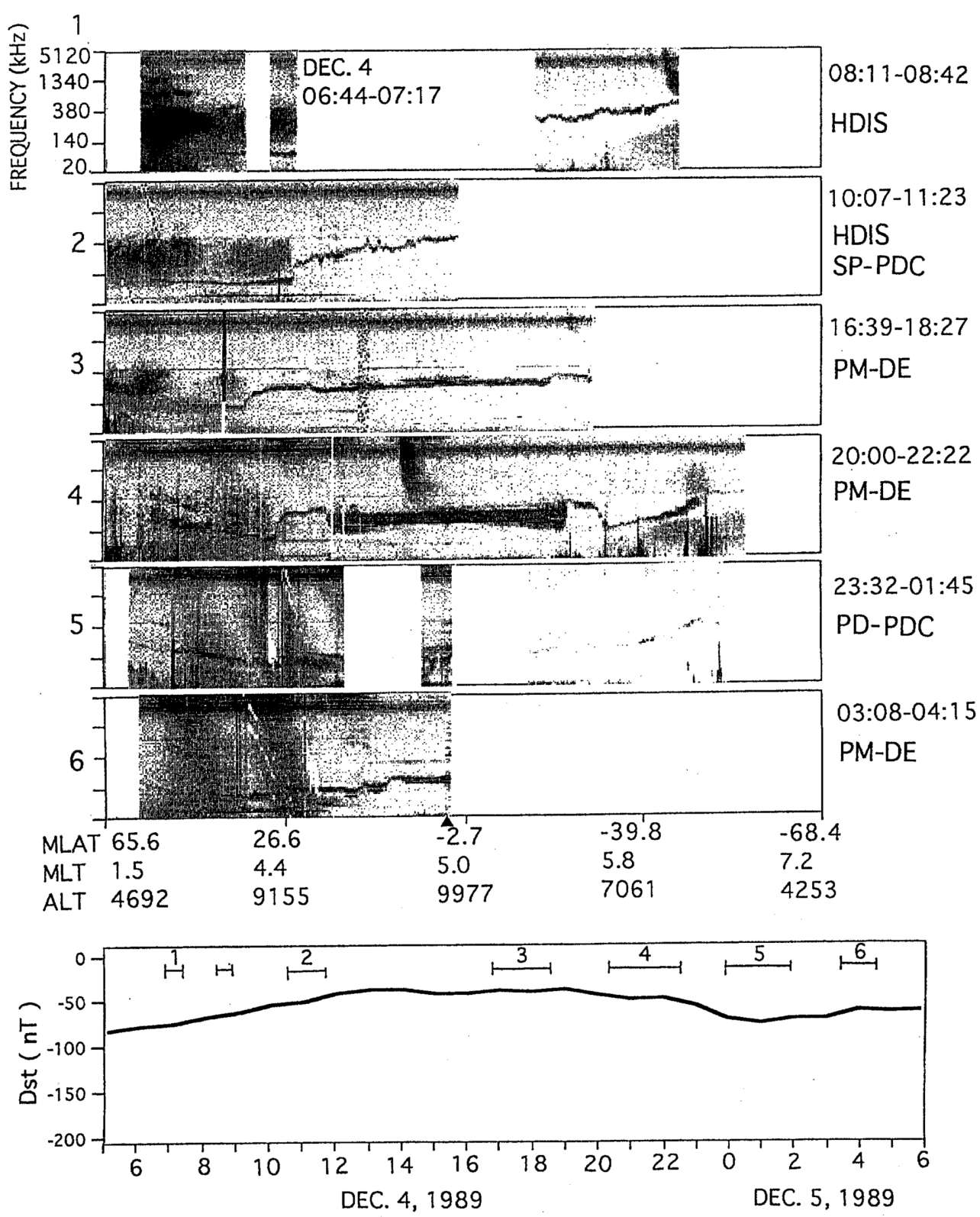

Fig. 5. Example series data of the dynamic spectra of PWS onboard the EXOS-D satellite for the observation in 1989 from $08: 11$ UT on Dec. 4 to 04:15 UT on Dec. 5, with Dst values covering the same period with PWS observations. In panel 3, the start of the donkey ears phenomena is recognized; the phenomena proceed showing typical "donkey ear" feature of PPDP along the satellite path as given in panel 4 , after 3 hours of the start of the phenomena. The entire plasmaspheric plasma evacuated in the period of panel 5. These series of dynamical variation of PPDP is intimately related to Dst variation: the periods corresponding to the panels 3 and 4 are understood as the DE-PDC related to $\partial D s t / \partial t>0$ about 10 hours earlier than the time of the occurrence of the donkey ears phenomena. The evacuating phenomena given in the panel 5 is PD-NDC; the phenomena are related to the period of $\partial D s t / \partial t<0$ that occurred about $2 \sim 3$ hour earlier than the occurrence of the phenomena. 


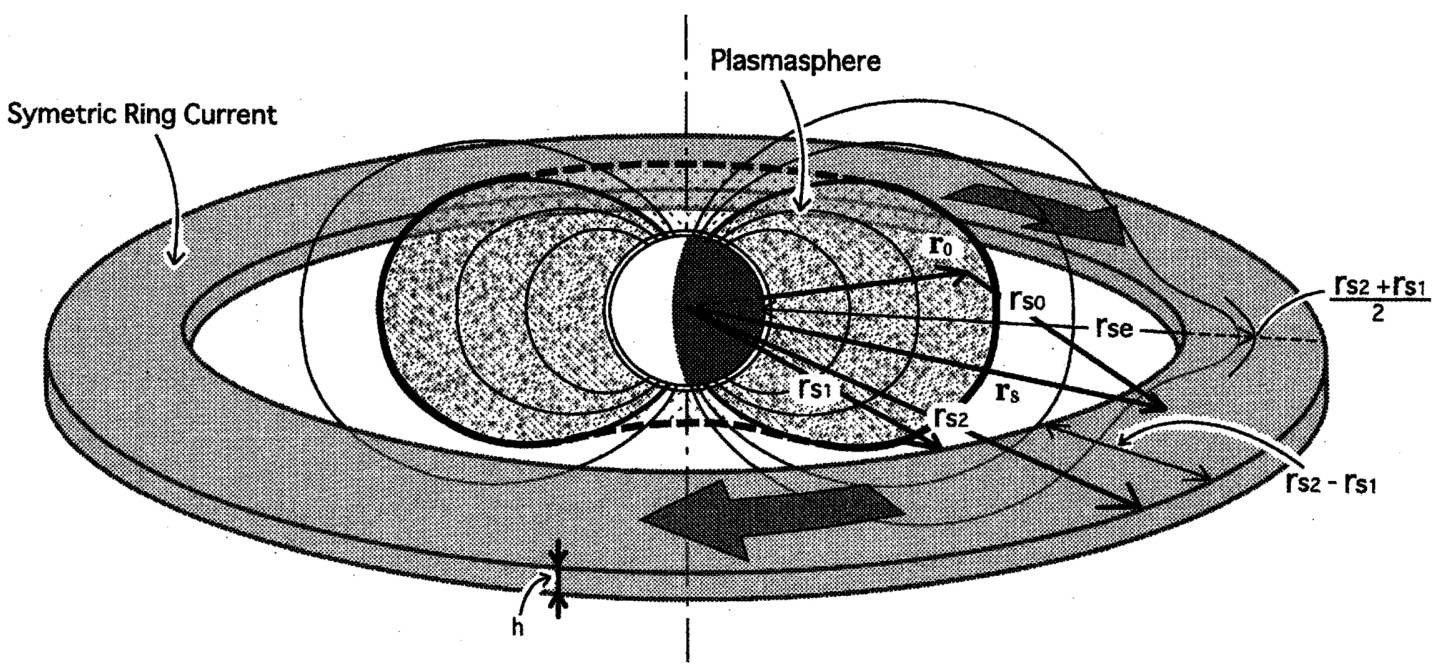

Fig. 6. Model of the symmetric ring current. Region of ring current pervades from $r_{s 1}$ to $r_{s 2}$ in radial direction with constant thickness of $h$, Magnetic field intensity at the position $r_{0}$ caused by the ring current can be expressed by using the $D s t$ that also can be expressed by the integration effects of ring current whose element is located at the distance of $r_{s e}$ (see Eqs. (10)(12)).

\section{Proposal of Betatron Plasma Drift}

We propose here a unified theory to interpret the plasmasphere behavior under the condition of the magnetic field variation in term of the plasma drift caused by the time varying electric field that is generated by the time dependent variation of the ring currents. Because the origin of the electric field is the same with the mechanism of the electric field generation in the betatron accelerator, we here call the process of present consideration betatron plasma drift. The basic equations are given for the generation of the electric field as

$$
\operatorname{rot} E=-\frac{\partial B}{\partial t}
$$

with

$$
\boldsymbol{B}=\operatorname{rot} \boldsymbol{A}
$$

where the vector potential $\boldsymbol{A}$ is expressed by the ring current $\boldsymbol{I}$ which is here simply given as the function of the distance measured from the center of the earth, as

$$
\boldsymbol{A}=\frac{\mu_{0}}{4 \pi} \int_{s} \frac{I\left(r_{s}\right)}{r_{s o}} d V_{s}
$$

In Eq. (3), $r_{s o}$ indicates the distance between the source point $r_{s}$ of the ring current and the observation point $r_{o}$

$$
r_{s o}=\left|r_{s}-r_{o}\right|
$$


and the integration is made for the entire source current region $s$ with the volume element $d V_{s}$ of the ring current.

When we substitute Eq. (2) into Eq. (1), with Eqs. (3) and (4), then, it follows that

$$
\operatorname{rot}\left\{\boldsymbol{E}+\frac{\mu_{0}}{4 \pi} \int_{s} \frac{\partial \boldsymbol{I}\left(r_{s}\right) / \partial t}{r_{s o}} d V_{s}\right\}=0
$$

This relation results in the electric field as

$$
\boldsymbol{E}=\boldsymbol{E}_{\text {out }}-\frac{\mu_{0}}{4 \pi} \int_{s} \frac{\partial \boldsymbol{I}\left(r_{s}\right) / \partial t}{r_{s o}} d V_{s}
$$

where $\boldsymbol{E}_{\text {out }}$ is the field impressed from outside and satisfies

$$
E_{\text {out }}=-\operatorname{grad} \phi
$$

for a given scalar function. For the electric field given by Eq. (6), then, the plasma drift velocity $V_{\mathrm{D}}$ is obtained as

$$
V_{\mathrm{D}}=V_{\mathrm{out}}-\frac{\mu_{0}}{4 \pi B_{\mathrm{ob}}^{2}}\left[\int_{s} \frac{\partial \boldsymbol{I}\left(r_{s}\right) / \partial t}{r_{s o}} d V_{s}\right] \times \boldsymbol{B}_{\mathrm{ob}}
$$

where

$$
\boldsymbol{V}_{\mathrm{out}}=\frac{\boldsymbol{E}_{\mathrm{out}} \times \boldsymbol{B}_{\mathrm{ob}}}{B_{\mathrm{ob}}^{2}}
$$

The velocity $\boldsymbol{V}_{\text {out }}$ is, therefore, caused by the electric field $\boldsymbol{E}_{\text {out }}$ penetrating into the plasmasphere. By ring current the magnetic field variation that is averaged at earth's surface as the Dst value takes place. That is

$$
\boldsymbol{B}=\frac{\mu_{0}}{4 \pi} \operatorname{rot}\left[\int_{s} \frac{I\left(r_{s}\right)}{r_{s e}} d V_{s}\right]
$$

or

$$
B=\frac{\mu_{0}}{4 \pi} \int_{s} \frac{\left(r_{s}-r_{e}\right) \times I\left(r_{s}\right)}{r_{s e}^{2}} d V_{s}
$$

for the ring current position at $\boldsymbol{r}_{s}$ and the observation position at $\boldsymbol{r}_{e}$ on the earth with $r_{s e}=\left|\boldsymbol{r}_{s}-\boldsymbol{r}_{e}\right|$. For the ring current, we here apply a simple model in which the current that starts from $r_{s}=r_{s 1}$ and end at $r_{s}=r_{s 2}$ with thickness of $h$. The region of ring current makes arc with azimuthal angle of $2 \pi \alpha$ (for $0<\alpha<1$, see Fig. 7). Because our main purpose here is to make dimension analyses to check quantitative validity of 


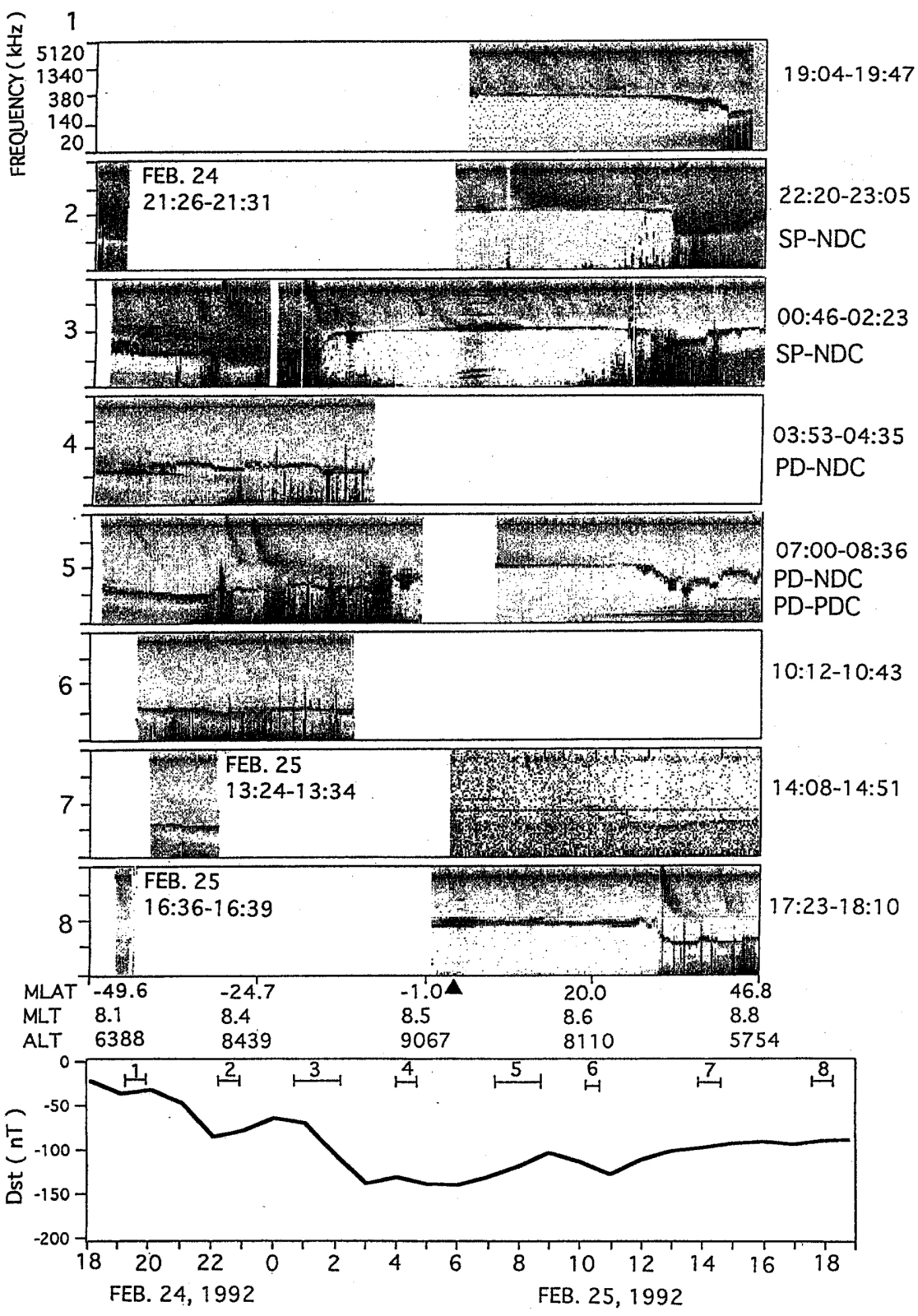

Fig. 7(a). Series of dynamic spectra of PWS onboard the EXOS-D satellite for the observation period from 19:04 UT on Feb. 24, 1992 to 18:10 UT on Feb. 25, 1992; the PWS dynamic spectra are indicated being divided into panels from 1 to 8 of corresponding satellite orbits. The Dst value is plotted in the bottom panel where the periods of the satellite observations of corresponding panels are indicated with the same number labeled in the panel. In the dynamic spectra given in the above panels are indicated versus the satellite position shown in the portion between the last dynamic spectra panel and the $D s t$ panel; a black triangle mark indicates the position of the magnetic equator and almost close to the apogee of the satellite orbit in these periods of observations. The ordinates of the dynamic spectra panels are the frequency; all tick marks in the ordinates indicate the same frequencies with the case of the top panel. In each panel of the dynamic spectra, the upper hybrid waves are indicated with the trend of continuous curve; from the upper hybrid frequency data $f_{\mathrm{UHR}}$, we can approximately obtain the electron density $N$ (that is, $f_{\mathrm{UHR}}$ is almost same with plasma frequency) as $N=f_{\mathrm{UHR}}{ }^{2} / 81$ (in the unit of $1 / \mathrm{cc}$ for $N$ and $\mathrm{kHz}$ for $f_{\mathrm{UHR}}$ ) in the region of high altitude higher than $6000 \mathrm{~km}$. The comments on the morphology of PPDP (plasmaspheric plasma density profile) are given in the right hand side of the corresponding panels using acronyms. 


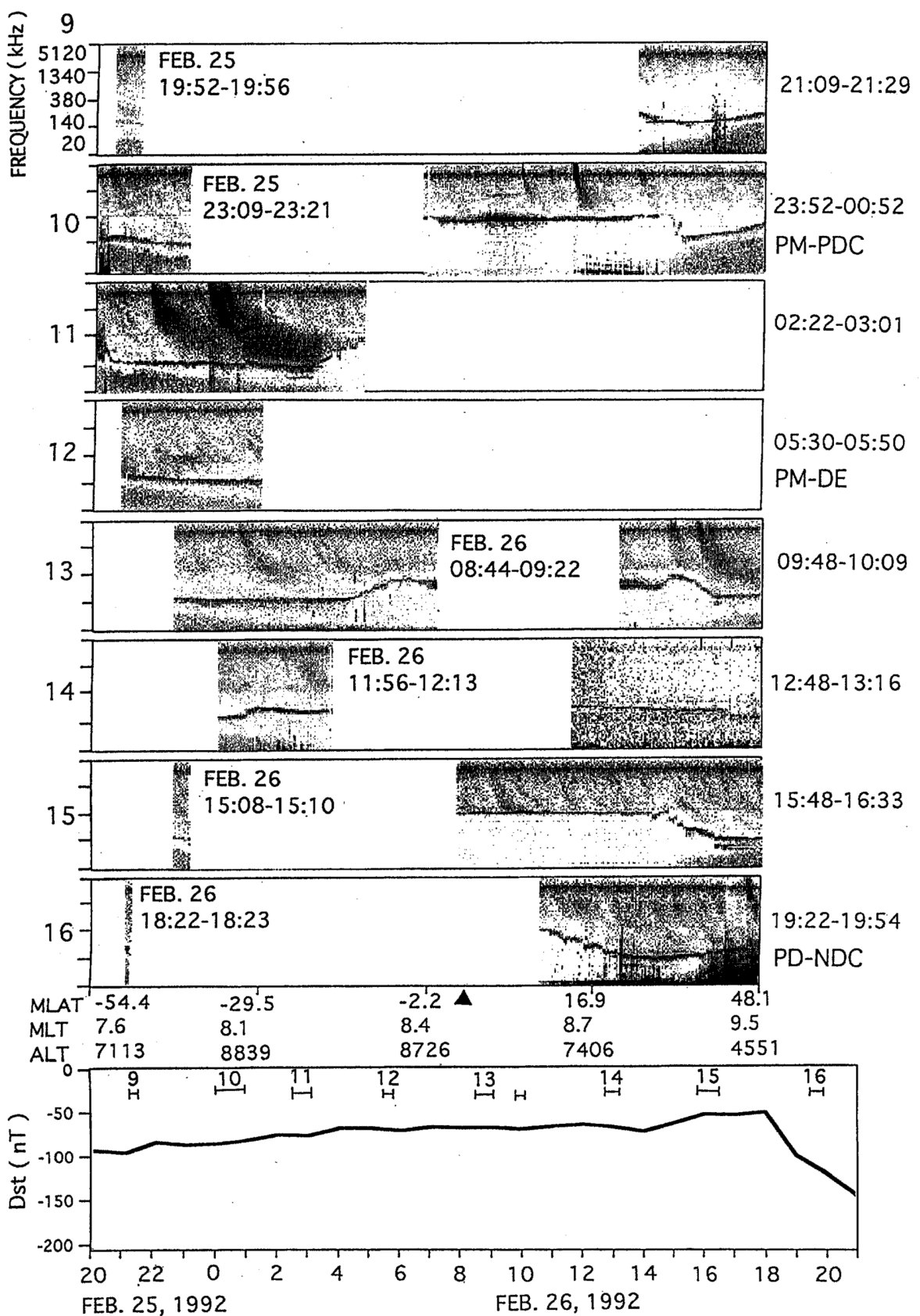

Fig. 7(b). Same with (a) for the period 19:52 UT on Feb. 25, 1992 to 19:54 UT on Feb. 26, 1992. The succeeding dynamic spectra are indicated being separated into panels 9 to 16 corresponding to revolutions of the satellite.

the betatron plasma drift motion, we also apply a crude approximation as given below, i.e.,

$$
D s t=\frac{\mu_{0}}{4 \pi} \frac{2 \pi \alpha h}{\bar{r}_{s e}^{2}} \cdot \int_{r_{s 1}}^{r_{s 2}} I\left(r_{s}\right) r_{s} d r_{s}
$$




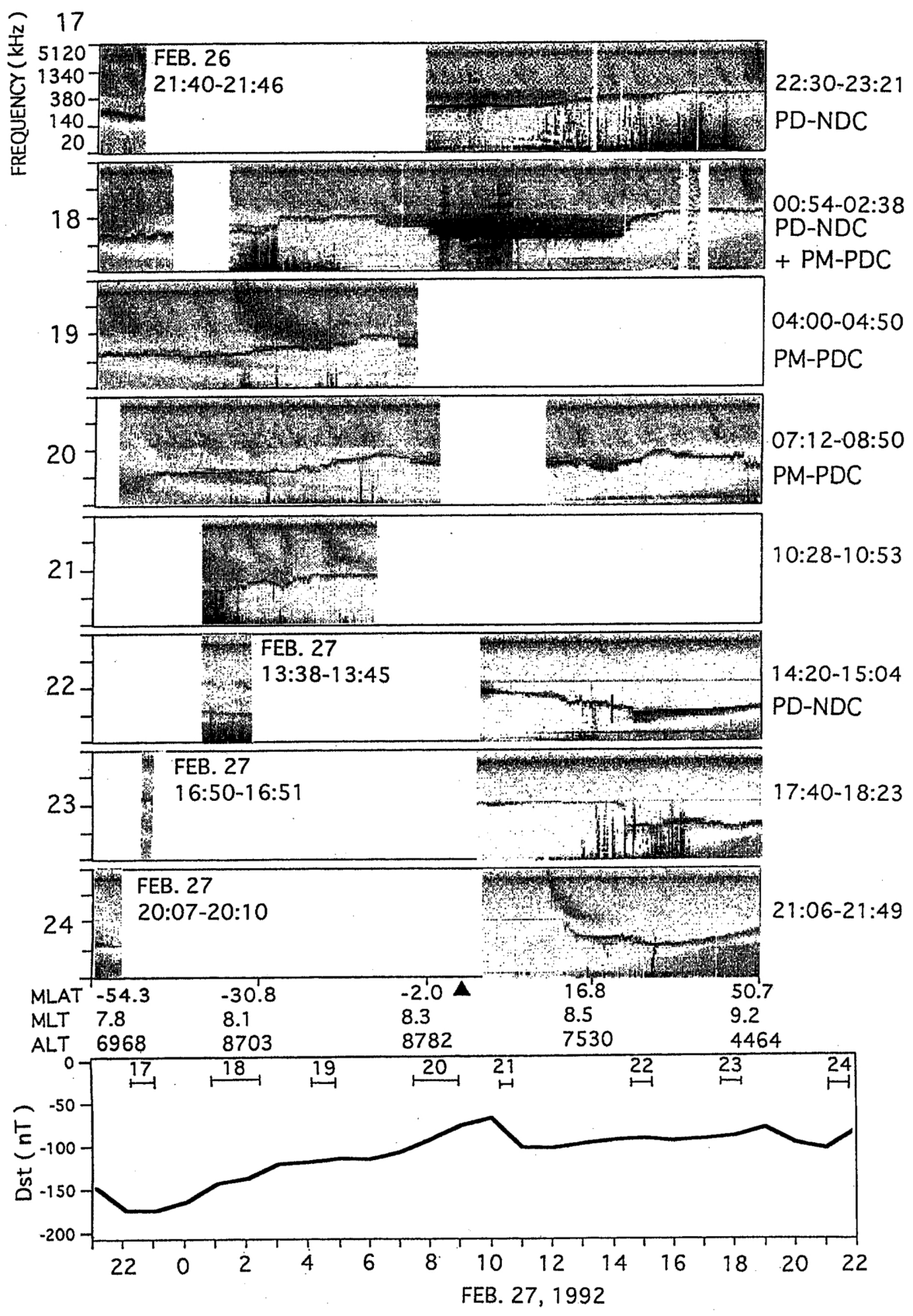

Fig. 7(c). Same with (a) for the period from 21:40 UT, on Feb. 26, 1992 to 21:49 UT on Feb. 27, 1992. The succeeding dynamic spectra are indicated being separated into the corresponding panels from 17 to 24 :

where $\bar{r}_{s e}$ shows an weighted average distance. Dst indicates the component of $\boldsymbol{B}$ which is considered to be approximated by Dst value observed on the earth's surface. For the same model of the ring current with same crude approximation, we can rewrite the betatron drift velocity $V_{\mathrm{D} \beta}$ in equatorial region, from the second terms of Eq. (8), as 


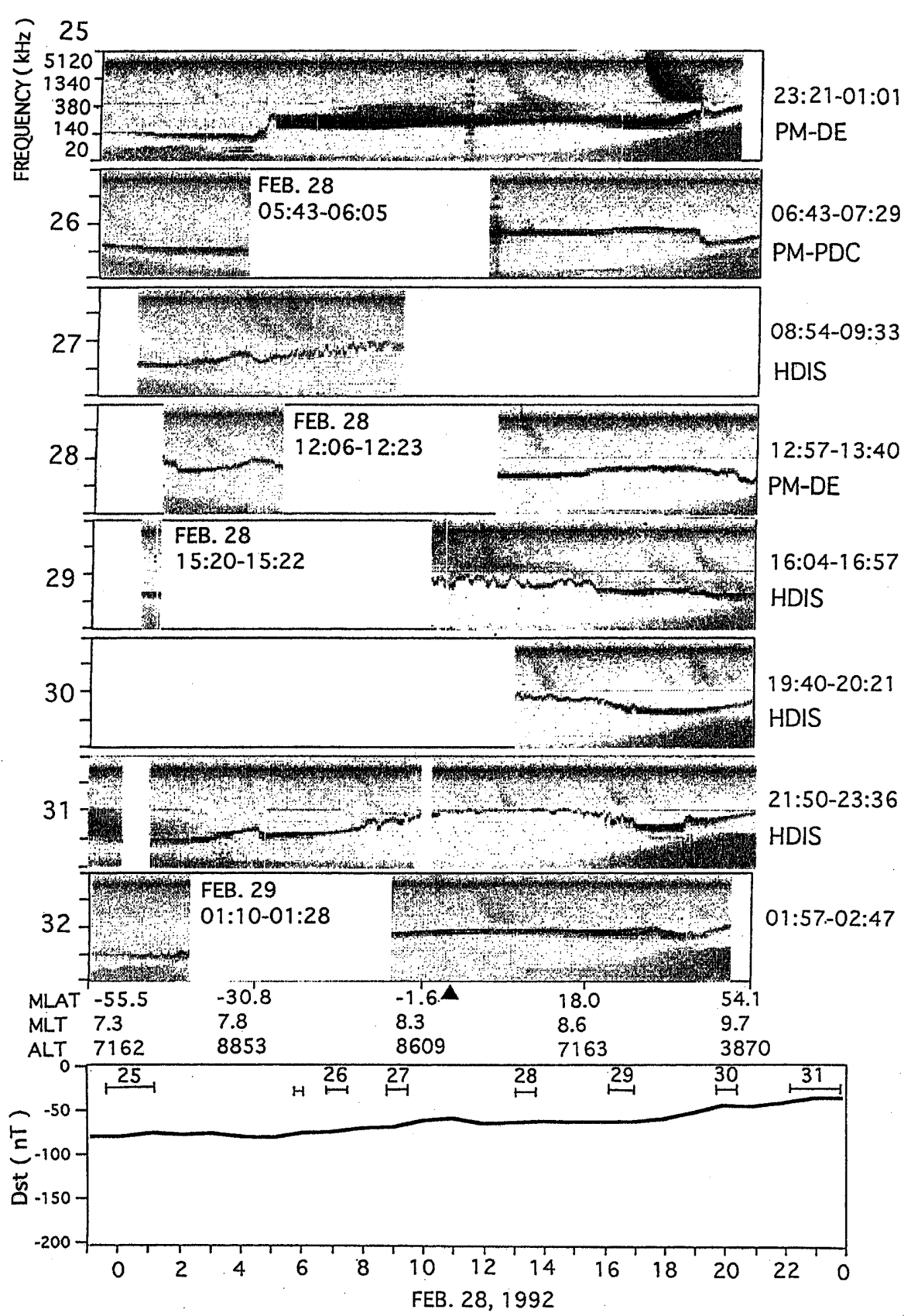

Fig. 7(d). Same with (a) for the period from 23:21 UT on Feb. 27, 1992 to 02:47 UT on Feb. 29, 1992. The succeeding dynamic spectra are indicated being separated into panels 25 to 32 corresponding to revolutions of the satellite.

$$
V_{\mathrm{D} \beta}=-\frac{\mu_{0}}{4 \pi B_{\mathrm{ob}}} \cdot \frac{2 \pi \alpha h}{\bar{r}_{s o}}\left[\int_{r_{s 1}}^{r_{s 2}}\left(\frac{\partial I}{\partial t}\right) r_{s} d r_{s}\right] \hat{r}
$$

where $\bar{r}_{s o}$ indicates the weighted average distance between the ring current and the observation point in 
the plasmasphere where the plasma is making the drift motion.

Using Dst value expressed in Eq. (12), then, the drift velocity is finally expressed by

$$
V_{\mathrm{D} \beta}=-\left(\frac{\bar{r}_{s e}^{2}}{\bar{r}_{s o}}\right) \frac{1}{B_{\mathrm{ob}}} \cdot \frac{\partial D s t}{\partial t} \hat{r}
$$

The result shows that in the period of negative $\partial D s t / \partial t$ value, plasma flows toward outside of the plasmasphere while the plasma flows into the plasmasphere from the inner magnetosphere in the case of the positive $\partial D s t / \partial t$ corresponding to the recovery phase of the magnetic storm. Assuming four example cases, the drift velocity is estimated as given in Table 1 . The estimated drift velocity is in the range from $100 \mathrm{~m} / \mathrm{sec}$, for weak magnetic storm, to $800 \mathrm{~m} / \mathrm{sec}$ for intense magnetic storm. The traveling time $T$ of the drifting plasma from the distance $r_{1}$ to $r_{2}$ can then be expressed for the inward flow or outward flow as

$$
T=\left|\int_{r_{1}}^{r_{2}} \frac{d r}{V_{\mathrm{D} \beta}}\right| .
$$

The expression in Eq. (15) is rewritten by using normalized quantities as

$$
T=\frac{1}{2} \frac{R_{s o}}{R_{s e}^{2}}\left|\frac{1}{\dot{D} s t}\left(\frac{1}{R_{2}^{2}}-\frac{1}{R_{1}^{2}}\right)\right|
$$

\begin{tabular}{|c|c|c|c|c|c|}
\hline \multicolumn{2}{|c|}{$\begin{array}{l}\text { Mean Ring Current } \\
\text { Position, } \bar{r}_{\mathrm{se}}\end{array}$} & \multicolumn{2}{|c|}{$4 r_{e}$} & \multicolumn{2}{|c|}{$6 r_{e}$} \\
\hline \multicolumn{2}{|c|}{$\begin{array}{l}\text { Location in the } \\
\text { Plasmasphere } \\
\text { given by the } \\
\text { Distance from the } \\
\text { Mean Ring Current } \\
\text { Position, } \bar{r}_{\text {so }}\end{array}$} & $2 r_{e}$ & $1 r_{e}$ & $3 r_{e}$ & $2 r_{e}$ \\
\hline \multicolumn{2}{|c|}{$\begin{array}{l}\text { Magnetic Field } \\
\text { Intensity at the } \\
\text { given Location, } \\
\mathrm{B}_{\mathrm{ob}}(\mathrm{nT})\end{array}$} & $3.75 \times 10^{3}$ & $1.11 \times 10^{3}$ & $1.11 \times 10^{3}$ & $4.69 \times 10^{2}$ \\
\hline \multirow{3}{*}{$\begin{array}{l}\text { Change Rate } \\
\text { of the Dst(nT) }\end{array}$} & $5 n T / h$ & - & - & $\begin{array}{l}96.0 \\
\mathrm{~m} / \mathrm{sec}\end{array}$ & $\begin{array}{l}339 \\
\mathrm{~m} / \mathrm{sec}\end{array}$ \\
\hline & $10 \mathrm{nT} / \mathrm{h}$ & - & $\begin{array}{l}254 \\
\mathrm{~m} / \mathrm{sec}\end{array}$ & $\begin{array}{l}192 \\
\mathrm{~m} / \mathrm{sec}\end{array}$ & - \\
\hline & $30 \mathrm{nT} / \mathrm{h}$ & $\begin{array}{l}114 \\
\mathrm{~m} / \mathrm{sec}\end{array}$ & $\begin{array}{l}762 \\
\mathrm{~m} / \mathrm{sec}\end{array}$ & - & - \\
\hline
\end{tabular}

Table 1. Drift velocity.

$r_{\mathrm{e}}$ : The earth radius. 
where

$$
\dot{D} s t=\frac{\partial}{\partial t}\left(\frac{D s t}{B_{0}}\right), \quad R_{1}=\frac{r_{1}}{r_{e}}, \quad R_{2}=\frac{r_{2}}{r_{e}}, \quad R_{s o}=\frac{\bar{r}_{s o}}{r_{e}}, \quad R_{s e}=\frac{\bar{r}_{s e}}{r_{e}} .
$$

To check quantitative reasoning to explain the observation results for the present discussing betatron drift mechanism we here select three cases; i.e.,

i) Weak storm time case where $R_{s e}=5$, with $\dot{D} s t=(5 \mathrm{nT} / 30,000 \mathrm{nT}) /$ hour and the plasma drifts inwards from $R_{2}=4$ to $R_{1}=2.5$, or drifts outwards from $R_{1}=2.5$ to $R_{2}=4$;

ii) Middle scale storm time case where $R_{s e}=4.5$, with $\dot{D} s t=10 \mathrm{nT} / 30,000 \mathrm{nT} / \mathrm{hour}$, and the plasma drifts inwards from $R_{2}=3.5$ to $R_{1}=2.5$ or drifts outwards from $R_{1}=2.5$ to $R_{2}=3.5$;

iii) Large magnetic storm time case where $R_{s e}=4$, with $\dot{D} s t=30 \mathrm{nT} / 30,000 \mathrm{nT} /$ hour, and the plasma drifts inwards from $R_{2}=3$ to $R_{1}=2.5$ or drifts outwards from $R_{1}=2.5$ to $R_{2}=3$.

In all of the above three cases $R_{\text {so }}$ is set approximately as

$$
R_{s o}=R_{s e}-\left(R_{1}+R_{2}\right) / 2
$$

The estimation of the transit time using Eq. (16) then results the values given in Table 2 . The estimated transit times well coincide with the observed delay times for the occurrence of the phenomena observed by the Akebono satellite. That is; for weak magnetic storms, with the time variation rate of $D s t$ is around $5 \mathrm{nT} / \mathrm{h}$, in the case of the magnetic storm on Dec. 4, 1989, we can see the delay of about $15 \mathrm{~h}$ for the occurrence of the donkey ears phenomena. As has already been pointed out, the delay does not mean the characteristic time of the variation of the plasma density; the evolution of the plasma density, after arrival

Table 2. Travelling time of drifting plasma.

\begin{tabular}{|l|c|c|c|}
\hline $\begin{array}{l}\text { Time varying rate } \\
\text { of Dst }\end{array}$ & $5 \mathrm{nT} / \mathrm{h}$ & $10 \mathrm{nT} / \mathrm{h}$ & $30 \mathrm{nT} / \mathrm{h}$ \\
\hline $\begin{array}{l}\text { Normalized mean } \\
\text { location of the } \\
\text { ring current, } \mathrm{R}_{\text {se }}\end{array}$ & 5 & 4.5 & 4 \\
\hline $\begin{array}{l}\text { Normalized position } \\
\text { of the apogee of } \\
\text { the Akebono } \\
\text { satellite, } \mathrm{R}_{1}\end{array}$ & 2.5 & 2.5 & 2.5 \\
\hline $\begin{array}{l}\text { Initial location of } \\
\text { the start of the } \\
\text { drift motion }\end{array}$ & 4 & 3.5 & 3 \\
\hline $\begin{array}{l}\text { Estimated transit } \\
\text { time }\end{array}$ & $\begin{array}{l}7.37 \times 10^{4} \mathrm{sec} \\
(20.5 \mathrm{~h})\end{array}$ & $\begin{array}{l}3.13 \times 10^{4} \mathrm{sec} \\
(8.7 \mathrm{~h})\end{array}$ & $\begin{array}{l}6.875 \times 10^{3} \mathrm{sec} \\
(1.9 \mathrm{~h})\end{array}$ \\
\hline
\end{tabular}


of the immigrating plasma, takes place during much shorter time. Growth of the donkey ears phenomena, in the case of Dec. 4, 1989, takes place within almost one revolution time of the Akebono satellite i.e. $3 \mathrm{~h}$.

\section{Morphology of Plasmasphere Variation}

The dynamical behavior reflected by changing features of the plasmaspheric plasma density profiles which have been studied through the analyses of the data of PWS onboard the Akebono satellite during three years from March 8, 1989 to April 30, 1992, can be categorized, considering the dependence on the magnetic field disturbances, as i) plasmaspheric plasma density profile (PPDP) during the negative time derivative-coefficient (NDC) of $D s t$; ii) PPDP during the positive time derivative-coefficient (PDC) of $D s t$; and iii) PPDP during no remarkable variation (NRV) of the magnetic field exceeding the threshold of changing rate of $3 \mathrm{nT} /$ hour.

The typical case of PPDP-NDC takes place in the main phase of the magnetic storms; the record of the maximum value during the analyzed period was $-111 \mathrm{nT} /$ hour in the time of the large magnetic storm during March 13 to 14,1989 . Usually the values are in an average range from -8 to $-50 \mathrm{nT} / \mathrm{hour}$. It is, however, clarified that PPDP's make dramatic change even there is no remarkable magnetic storm, with low $K p$ index condition, if there are $D s t$ variations with time-derivative larger than $-5 \mathrm{nT} / \mathrm{hour}$.

The typical case of PPDP-PDC takes place in the recovery phase of the magnetic storms; the record of the maximum value of the time-derivative of PDC, is $125 \mathrm{nT} /$ hour that had happened also in the case of the large magnetic storm of March 13-14, 1989. As the case of PPDP-NDC, PPDP-PDC effects are not restricted in the storm times; the plasmaspheric plasma density profiles make sensitive response even in the calm magnetic field condition when there is temporary variation of the magnetic field exceeding the threshold of $5 \mathrm{nT} /$ hour.

Considering these natures of the variations of PPDP's therefore, we should clarify that under which circumstances of the time derivative-coefficient of Dst among NDC, PDC, and NRV, a specific phenomenon of PPDP variation occurs. Then dynamical behavior can be described by separating into following five morphological types:

type a. Plasmapause disruption under NDC states of Dst (PD-NDC): Remarkable effects take place in the mainphase of the magnetic storm; PD-NDC also takes place temporarily even in quiet conditions, in terms of $K p$, whenever NDC condition with rate larger than $5 \mathrm{nT} / \mathrm{h}$ occurs.

type b. Plasmapause modification under PDC states of Dst (PM-PDC): Remarkable effects take place in the recovery phase of the magnetic storm showing the donkey ears phenomena (PM-DE); but the same effects take place even under non storm condition whenever PDC condition with rate larger than 5 nT/h occurs.

type c. Steepening of cliff structure of the plasmapause under the NDC-condition (SP-NDC).

type d. Steepening of cliff structure of plasmapause under the PDC condition (SP-PDC).

type e. Highly disturbed irregular situation (HDIS): The characteristic points of this situation are in the oscillatory nature of the plasma density distribution profile revealed when the satellite traverses across the magnetic fields in the latitudinal direction; with scale lengths of 1000-3000 km the plasma density varies with amplitudes that are 2 to 4 times or $1 / 2$ to $1 / 4$ of the average plasma density values. This highly disturbed irregular situations frequently occur after the large magnetic storms following the donkey ears phenomena. There are however mysterious phenomena where the highly disturbed irregular situation of plasmasphere take place even under the dead calm situation (NRV) of the magnetic field activities (HDIS-NRV).

In Figs. 7(a) to 7(d) a series of PWS data observed from 19:00 UT on Feb. 24, 1992, to 02:58 UT on Feb. 29, 1992 are indicated; PWS data for the upper hybrid wave frequency are noted as relative indicator of the plasma density as the function of the satellite position taking the magnetic equator at the center of each panel. Though there are negligibly slight shift in the latitudinal values at given instances of abscissa between corresponding panels, the satellite position in term of the latitude is almost same for all panels. 
In each panel relative values of the plasma density variation can obtain from the upper hybrid frequency (UHR). Near the equator, the electron cyclotron frequency is less than $80 \mathrm{kHz}$ in the altitude range around $10,000 \mathrm{~km}$, then we can roughly estimate the plasma density directly from the indicated UHR frequency. That is, $340 \mathrm{kHz}$ gives $1782 / \mathrm{cc}$ and $140 \mathrm{kHz}$ gives $241 / \mathrm{cc}$. We can, therefore, see a very dramatic change of the plasma density profiles (PPDP) by tracing the variation of UHR from the panel 1 in Fig. 7(a) to the panel 32 in Fig. 7(d); sometimes, PPDP changes one order of magnitude within a few hours.

In each figure, the Dst values are given in the bottom panels, for each corresponding period, where hourly values of Dst are given; the observation period of the Akebono satellite is given in each corresponding times in the Dst diagram using the corresponding panel numbers of PWS data given in the indicated panels of the same figure. From Dst variation, we can determine the periods of NDC and PDC for corresponding PWS observations; but it should be noted that there are apparent delay time from a few hours to a few 10 hours to identify the corresponding phenomena of PPDP variations.

From panels 1 to 5 in Fig. 7(a), we can see NDC effects. In the initial phase, there is apparent signature of the steepening of cliff feature of plasmapause plasma density profile (SP-NDC) which suggests the enhancement of plasma convection just outside of the plasmapause. However, plasmapause destruction (PD-NDC) take place in the end period of the main phase of the magnetic storm. The plasma in the plasmasphere starts the exodus towards the plasma sheet region. This PD-NDC phenomena continue at least about 4 hours after the end of the main phase of the magnetic storm suggesting 4 hours delay of the response; this time delay can be interpreted as the traverse time of the outflowing plasma from starting region inside of the plasmasphere to the outside region of the plasmapause. In panel 17, we can see a dramatical feature of the plasmapause disappearance as result of the NDC processes where the plasmapause and outer plasmasphere have been completely disrupted.

When the magnetic storm has entered into the recovery phase, the PM-PDC phenomena occur; there are a few hour ( 3 hours in this case) delay after starting of the recovery phase, for appearing the feature of PDC effects. We can see continuation of phenomena in panels 18 to 20 where progress of the donkey ears phenomena (PM-DE) can be recognized. In this processes, the hot plasma from the plasma sheet makes immigration into the low latitude region centered around the magnetic equator. After 21:00 UT, Feb. 27, the geomagnetic condition enters into very long and gradual recovery phase where timederivative of Dst shows $2 \mathrm{nT} /$ hour during 24 hour period. In the last part of long recovery phase of the magnetic storm PPDP shows oscillatory characteristics that is here called highly disturbed irregular situation (HDIS-NRV), as they have been given in panel 27, and from panel 29 to panel 31.

\section{Local Time Dependence}

The local time dependence of occurrence characteristics of the PPDP morphological types among the phenomena of PD-NDC, PD-PDC, PM-NDC, PM-PDC, PM-DE, and HIDC are investigated for three years data starting from March 8, 1989 to March 10, 1992. Because the orbit of the Akebono satellite is highly eccentric with orbital inclination of about $60^{\circ}$, the latitudinal position of the apogee varies with period of about a year and five months. During presently investigated three years period, then, 2 sweeps of the local time survey in the altitude range from $4000 \mathrm{~km}$ to $9000 \mathrm{~km}$ in the plasmasphere have been made.

The results of analyses show that there is no special local time prohibiting occurrence of the presently discussing betatron plasma drift in the plasmasphere. This evidence also supports that the plasma movements associated with magnetic variation is not controlled by the plasma convection, in the inner magnetosphere, that shows intimate relation to the local time. We can then state that the plasmasphere are not simply controlled by the magnetospheric plasma convection and filling processes of the magnetic flux tube by the plasma from the ionosphere origin; but the plasma in the plasmasphere is dynamically controlled by the drift motions across the magnetic fields in the plasmasphere caused by the electric field being induced by the time dependent variations of the ring current.

In the storm time of the plasmasphere, therefore, principal variations of PPDP are controlled by the 
magnetic field variations; a large volume of the mass and energy are then exchanged directly between the inner magnetosphere and the plasmasphere.

\section{Discussions}

When we investigate the series of the data obtained by succeeding revolutions of the Akebono satellite, it is apparent that PPDP (plasmaspheric plasma density profile) shows extraordinarily dynamical behavior, even in the midst region of the plasmasphere around $L=2.5$ where the apogee of the Akebono satellite passes through. Principal causes of the dynamical feature is understood by the betatron plasma drift consistently, that is given by the second term of Eq. (8), and Eqs. (13) and (14). Because of $\boldsymbol{E} \times \boldsymbol{B}$ drift effect, the plasma can freely move across the magnetic field. The estimated values which are given in Table 1 for the drift velocity and in Table 2 for the transit time from the plasmapause to $L=2.5$ or visversa consistently explain the delay time obtained from the series of the data given in Figs. 7 (a)-7(d).

The density depression that takes place in period of the donkey ears phenomena (PM-DE) is here explained as the results of the injection of the hot but tenuous plasma from the plasma sheet. This conclusion is supported by the sharp change of the plasma density, along the same magnetic field lines in the plasmasphere, that show the contact surface between two different kinds of plasma. Because the hydrostatic quasi-equilibrium state along the magnetic field can only be maintained by the shock like pressure gradient between the plasmaspheric plasma (1) and immigrating plasma (2),

$$
\nabla P_{1}=\nabla P_{2}
$$

provides the condition

$$
n_{1} \kappa T_{1}=n_{2} \kappa T_{2}
$$

at the contact surface, where $P, n$ and $T$ are pressure, plasma number density and temperature, respectively. The depleted region plasma density $n_{2}$ is almost one order of magnitude lower than the plasmaspheric plasma density $n_{1}$. This conclude that two completely different origin of plasmas contact each other. Only the possible origin of the plasma with temperature which is one order of magnitude higher than plasmaspheric plasma temperature is in the outside region of the plasmasphere, mostly in the plasma sheet. When the feature of magnetic fields variation is switched from PDC state to NDC state, the plasma start to flow out then donkey ears phenomena disappear in a short time of period, say within 1 hour. We can see the example case on Feb. 27 to 29, on the other hand, where the plasma states change into high instability when the recovery phase of magnetic storm enters into a calm condition. Because the electric fields controlling the plasma drift disappear, in this period, the plasma enters the state of interchange instabilities at the portion of the contact of "cold and dense" plasmaspheric plasma with "hot and tenuous" plasma originated from the plasma sheet.

At this stage of discussion, a question is raised; is the previous concept of the plasmasphere and plasmapause formation not correct? or how the present concept of the betatron plasma drift is related to the control of plasma convection? As answer we can simply show expressions of Eqs. (6) and (8) as general expressions of the electric fields and resulted plasma motions. Plasma in the plasmasphere is subjected to the drift due to electric field $\boldsymbol{E}_{\text {out }}$ that is formed by the relative motion of the corotating frame with respect to the plasma convection. In addition to the effect of $\boldsymbol{E}_{\text {out }}$, therefore, the induction field effects is superposed. When the time dependent effect is smaller, then, the plasmapause formation is controlled by the convection field in the plasma sheet. When the induction field term overcomes the effect of the convection field, however, plasmapause formation and feature of the plasmasphere variation are controlled by the betatron plasma drift. An example of transition of the plasmasphere variation process can be seen in the data of the Akebono satellite observed on Feb.26, 1992. In the very early time of the main phase of the magnetic storms, when the majority of plasmas flowing outwards do not arrive at the 
plasmapause, the effect of the enhancement of the plasma convection makes the plasmapause cliff steep; but soon after the feature of steepening plasmapause is destroyed due to outwards flow of plasma, resulting extraordinarily decreased plasma density in the outer region of the plasmasphere.

\section{Conclusion}

From the upper hybrid wave frequency detected from PWS data onboard the Akebono (EXOS-D) satellite, the plasmaspheric plasma density profiles (PPDP) have been analyzed continuously. The PPDP variation reveals extremely dynamical features that sensitively reflect the effects of magnetic field variations; these PPDP variations are classified into two major categories which take place in the positive phase of the time derivative-coefficient of the magnetic field (PDC: $\partial D s t / \partial t>0$ ) and the negative phase of the time derivative-coefficient (NDC: $\partial D s t / \partial t<0$ ). Remarkable PDC states occur corresponding to the recovery phase of the magnetic storms while NDC states occur corresponding to the initial and main phases of the magnetic storms. Not necessarily being related to the magnetic storm times, however, these PPDP variation phenomena take places in the every PDC, and NDC phases of the time variation of the magnetic field when the time derivative-coefficient exceeds I $5 \mathrm{nT} / \mathrm{h}$ I.

In the NDC phases, typical PPDP variation is characterized by following: i) Initially, reduction of plasmasphere size occur with sharpening of the cliff structure (SP-NDC) of PPDP; and then ii) disruption of the plasmapause (PD-NDC) take place with expansion of the plasmasphere size while the plasmaspheric plasma density is reduced mostly by $1 / 5$ to $1 / 10$. The phenomena of SP-NDC occur in the very beginning of the main phase of the magnetic storms but the phenomena are taken over by PD-NDC.

In the PDC phase, PPDP variation is characterized by the modification of the plasmapause by reducing the plasma density from the region of the magnetic equator. The phenomena are called donkey ears phenomena (DE-PDC); the PPDP in these phenomena is characterized by discontinuity of the plasma density along the same magnetic field lines having the high density part in the high latitude side. All these dynamical variations of the PPDP associated with PDC, and NDC phases of the geomagnetic field variation are concluded as to be caused by the $\boldsymbol{E} \times \boldsymbol{B}$ drift of the plasma. The electric fields consist of two components; these are, the field transported from plasma convection region located in the plasma sheet and the induction field caused by time variation of the ring current which result in $\partial B / \partial t$; the later case is named as betatron drift in this paper. Though SP-PDC, or SP-NDC phenomena are related to the enhancement of plasma convection in the early phase of the magnetic storm, such convection effects are taken over by the increment of the induction electric fields. The plasmaspheric plasmas make exodus towards the plasma sheet region in the time of negative time derivative-coefficient of the geomagnetic field, showing the characteristics of PD-NDC phenomena. The hot plasma in the plasma sheet immigrate into the plasmasphere due to the drift motion starting from the region of the plasmapause around the magnetic equator. Through this immigrating phase of the plasma sheet plasma into the plasmasphere, a quasi-equilibrium state of the pressure balance is formed at contact surface that are formed across the magnetic field lines in the perpendicular direction of the field. That is, the shock-like sharp boundary is formed at the contact surface between the cold plasmaspheric plasma and hot plasma from the plasma sheet origin with the state of sharp plasma density variation at the contact surface also.

The estimation of the plasma drift velocities in the plasmasphere around $3 \sim 4 R_{\mathrm{e}}$ due to the induction field effects are in the range from $70 \mathrm{~m} / \mathrm{sec}$ to $700 \mathrm{~m} / \mathrm{sec}$ corresponding the time derivative-coefficient of the geomagnetic fields observed at the earth's surface respectively from $5 \mathrm{nT} /$ hour to $30 \mathrm{nT} / \mathrm{hour}$. Because of the traverse time of the drifting plasma, arrival of the hot plasma from the plasma sheet is always delayed in a range from 15 hours (for $5 \mathrm{nT} /$ hour case) to 2.5 hours (for $30 \mathrm{nT} /$ hour case) to detect the arrival of the hot plasma at the satellite level around 2.5 earth radii in the plasmasphere. The occurrence of the characteristic PPDP phenomena including the variation of the plasmapause structure is observable for all local time as this has been confirmed through entire three years data of PWS observations of the Akebono satellite.

All of these evidences of PPDP variation verify the betatron plasma drift and give conclusion that 
the previous paradigm of the plasmasphere physics should be rewritten by new paradigm based on the concept of this betatron plasma drift. That is, the plasmasphere is not the region of diffusive equilibrium where only the plasma sheet plasma convection and the filling processes from the ionosphere are significant, and is not the plasma region separated from the inner magnetosphere forming the plasmapause but is the region of very active and dynamic behavior where large amount of plasma energy and mass pass through being coupled with mass transfer between the inner plasma sheet and the topside ionosphere. Plasmapause is, therefore, no more constant existence caused by the differential motion between the corotating region of the plasmasphere and convection region of the inner plasma sheet, but the plasmapause is frequently disrupted by the exodus of plasmaspheric plasma into the plasma sheet in the negative phase of the time variation of the geomagnetic field and also by the immigration of hot plasma into the plasmasphere from the neighboring plasma sheet.

The present works are related to the international collaborating program "Solar Terrestrial Energy Program" (STEP) which implementation in Japan is supported by the ministry of education, science and culture. The present studies are mainly based on the PWS observation data onboard the Akebono (EXOS-D) satellite; the author is very much grateful to the PWS team member Prof. A. Morioka. The arrangement of PWS data have been carried out by Dr. lizima; the author is also grateful for his extensive assistance.

\section{REFERENCES}

Angerami, J. J. and J. O. Thomas, Studies of planetary atmosphere: 1. The distribution of electrons and ions in the earth's exosphere, J. Geophys. Res., 69, 4537-4560, 1964.

Axford, W. I. and C. O. Hines, A unifying theory of high latitude geophysical phenomena and geomagnetic storms, Can. J. Phys., 39, 1433-1464, 1961.

Brice, N. M., Bulk motion of the magnetosphere, J. Geophys. Res., 72, 5193-5211, 1967.

Carpenter, D. L., Whistler evidence of a 'knee' in the magnetospheric ionization density profile, J. Geophys. Res., 68, 1675-1682, 1963.

Chappel, C. R., K. K. Harris, and G. W. Sharp, A study of the influence of magnetic activity on the location of the plasmapause as measured by OGO 5, J. Geophys. Res., 75, 50-56, 1970.

Nishida, A., Formation of plasmapause, or magnetospheric plasma knee, by the combined action of magnetospheric convection and plasma escape from the tail, J. Geophys. Res., 71, 5669-5679, 1966.

Oya, H., Studies on plasma wave in the plasmasphere and auroral particle acceleration region, by PWS onboard the EXOS-D (Akebono) satellite, J. Geomag. Geoelectr., 43, 369-393, 1991.

Oya, H., A. Morioka, K. Kobayashi, M. Iizima, T. Ono, H. Miyaoka, T. Okada, and T. Obara, Plasma wave observation and sounder experiments (PWS) using the Akebono (EXOS-D) satellite-Instrumentation and initial results including discovery of the high altitude equatorial plasma turbulence, J. Geomag. Geoelectr., 42, 411-442, 1990.

Singh, N. and J. L. Horwitz, Plasmasphere refilling: recent observations and modeling, J. Geophys. Res., 97, $1049-1079,1992$. 